\title{
Estrategia Ludiproblemas para mejorar la competencia de resolución de problemas matemáticos
}

\author{
Percy Fredy Huanacuni Huanacuni \\ percyhuanacuni20@gmail.com \\ Universidad Nacional Pedro Ruiz Gallo \\ Esther Ruth Mamani Chipana \\ miss.ruty.7@gmail.com \\ Universidad Nacional Pedro Ruiz Gallo \\ Sara Virginia Huanacuni Huanacuni \\ huanacunisara2@gmail.com \\ Universidad Nacional Pedro Ruiz Gallo \\ Perú
}

\section{RESUMEN}

En los últimos años el aprendizaje en el área de matemática está basado en el desarrollo de distintos conocimientos sin buscar el desarrollo de la competencia en la resolución de problemas, en la escuela los estudiantes necesitan de un ambiente donde formulen y validen conjeturas, se planteen preguntas, utilicen procedimientos propios, manipulando objetos concretos y adquieran las herramientas necesarias. Por tal motivo se planteó como objetivo, aplicar la Estrategia "LUDIPROBLEMAS" para elevar la competencia de resolución de problemas matemáticos en los estudiantes, formando así personas capaces de solucionar problemas de la vida diaria. El tipo de investigación es aplicada, que tiene por finalidad de obtener conocimientos y solucionar problemas que se desarrolla mediante un proceso. Con un enfoque cuantitativo. Se manejó una muestra poblacional de 24 estudiantes. El resultado de la investigación indica que el 67\% de los estudiantes del grupo de aplicación se encuentra en el nivel de logro previsto, logrando así elevar la competencia de resolución de problemas con la aplicación de las actividades de la estrategia LUDIPROBLEMAS.

Palabras clave: estrategia ludiproblemas; problemas matemáticos; competencia. 


\title{
Strategy Ludiproblemas to improve the competence of solving mathematical problems
}

\begin{abstract}
In recent years learning in the area of mathematics is based on the development of different knowledge without seeking the development of competence in problem solving, at school students need an environment where they formulate and validate conjectures, ask questions, use their own procedures, manipulating concrete objects and acquire the necessary tools. For this reason, the objective was to apply the "LUDIPROBLEMS" Strategy to raise the competence of solving mathematical problems in students, thus training people capable of solving problems in daily life. The type of research is applied, which aims to obtain knowledge and solve problems that are developed through a process. With a quantitative approach. A population sample of 24 students was handled. The result of the research indicates that $67 \%$ of the students in the application group are at the expected level of achievement, thus achieving raising the problem-solving competence with the application of the activities of the LUDIPROBLEMS strategy.
\end{abstract}

Keywords: ludiproblems strategy; mathematical problems; competence.

Artículo recibido: 30 noviembre. 2021 Aceptado para publicación: 29 diciembre 2021 Correspondencia: percyhuanacuni20@gmail.com Conflictos de Interés: Ninguna que declarar

1. 
Huanacuni Huanacuni y otros

Ciencia Latina Revista Científica Multidisciplinar, Ciudad de México, México. ISN 2707-2207 / ISSN 2707-2215 (en línea), enero- febrero, 2022, Volumen 6, Número 1. https://doi.org/10.37811/cl_rcm.v5i4.683 p1668 


\section{INTRODUCCIÓN}

La Organización de las Naciones Unidas para la Educación, la Ciencia y la Cultura (UNESCO), a través del Programa para la evaluación Internacional de Estudiantes (PISA) informa que los estudiantes tienen resultados bajos en lo que respecta al aprendizaje del área de matemática, mostrando un bajo nivel de desempeño en la resolución de problemas, serias dificultades para traducir y expresar matemáticamente las condiciones propuestas en situaciones problemáticas. Esta evaluación se aplica cada tres años e incluye las áreas de Comprensión lectora, Matemática y Ciencias. En cada ciclo se hace énfasis en una de las áreas mencionadas.

Uno de los problemas que atraviesa actualmente el Perú, es la crisis en la educación especialmente en la enseñanza aprendizaje de la matemática.

Una de las causas es que el docente no desarrolla los procesos metodológicos de la matemática que comienza con la manipulación de objetos concretos, los gráficos o imágenes y finalmente lo simbólico o lo abstracto.

Otra de las causas es la escasa aplicación de materiales lúdicos, ejercicios atractivos con imágenes, y recursos tecnológicos, en el proceso de enseñanza-aprendizaje causando baja motivación durante el desarrollo de aprendizaje de los estudiantes.

La principal causa es la insuficiente aplicación de estrategias por parte del docente, que motive al estudiante a desarrollar problemas y ejercicios contextualizados a la realidad del estudiante que ayude a desarrollar el pensamiento de resolver problemas de la vida diaria, formándolo como una persona proactiva para la sociedad peruana.

La manera de definir la matemática cambia constantemente, como es de verse, cada generación lo adecua de acuerdo a la situación en la que se utiliza, según el Ministerio de Educación (2006) cita las siguientes definiciones: "Para los griegos, la matemática era la ciencia de la cantidad y del espacio. Las ciencias de la cantidad y del espacio eran, obviamente, la aritmética y la geometría." (P.7)

Una idea de Descartes (Siglo XVII) leída en una publicación del Ministerio de Educación (2006) menciona que la matemática "es la ciencia del orden y la medida..." (p. 8)

Por otro lado Guzmán (2007) define la matemática de la siguiente manera: "Es una ciencia interesante dinámica y cambiante: de manera rápida y turbulenta en sus propios contenidos y aún en su propia concepción profunda...” (p. 21) 
Así mismo, la matemática es la ciencia de la cantidad, del orden, de la medida, dinámica y cambiante, al pensarlo uno se educa, se entrena y se prepara porque es posible que vuelvan a aparecer en la vida cotidiana usando disfraces.

El área de matemática es uno de las áreas con mayores horas en la educación peruana y la más preocupante en el rendimiento académico. Según el Ministerio de Educación (2010, p. 47) "Las capacidades explícitas en el área de matemática involucra los procesos transversales de razonamiento y demostración, Comunicación Matemática y resoluciones de matemáticas, siendo este último el proceso a partir del cual se formulan las competencias del área."

Así mismo, en la reflexión sobre la concepción de la matemática surgen opiniones, aportes y filosofías sobre la actividad matemática y la capacidad de aprender la matemática según Alcalá y otros(2006)mencionan que: "La matemática actual no solo pretende resolver los mismos problemas que la matemática de toda la vida, sino que no quiere desentenderse de ninguna de las que se presenten en la vida cotidiana, aunque no pueda ofrecer soluciones exactas.” (p. 17)

De este modo el área de matemática es una disciplina provista de actitudes, contenidos, capacidades y competencias, involucrando los procesos transversales de razonamiento y demostración, comunicación matemática y resoluciones matemáticas, que pretende resolver los mismos problemas que se presenten en la vida cotidiana.

Desde mucho tiempo se realiza el estudio de objetos, siendo importante el estudio del área de matemática para las personas según el Ministerio de Educación en el Diseño Curricular Nacional (2009, pag.186) el Área de Matemática se fundamenta en: La matemática forma parte del pensamiento humano y se va estructurando desde los primeros años de vida en forma gradual y sistemática, a través de las interacciones cotidianas. Los niños observan y exploran su entorno inmediato y los objetos que lo configuran, estableciendo relaciones entre ellos cuando realizan actividades concretas de diferentes maneras: utilizando materiales, participando en juegos didácticos y en actividades productivas familiares, elaborando esquemas, gráficos, dibujos, entre otros. Así mismo la Matemática está presente en el proceso educativo para contribuir al desarrollo según Acevedo(2007, pág. 6) menciona que: La escuela debe preparar a los alumnos para ser ciudadanos productivos y en consecuencia, además de que la formación matemática es un requisito esencial para el estudio de una amplia variedad de disciplinas, 
debe potenciar a los estudiantes con los conocimientos, destrezas y formas de razonamiento que requieran para su vida diaria.

Por lo que es importante el Área de Matemática durante los primeros años de vida a través de las interacciones cotidianas, los niños exploran su entorno cuando realizan actividades concretas, desarrollando las destrezas necesarias para resolver problemas cotidianos, el Área de Matemática debe potenciar a los estudiantes con los conocimientos, destrezas y formas de razonamiento que requieran para su vida diaria.

Las capacidades explícitas para cada grado involucran los procesos transversales de Razonamiento y demostración, Comunicación matemática y Resolución de problemas, siendo este último el proceso a partir del cual se formulan las competencias del área en los tres niveles, según el Ministerio de Educación en el Diseño Curricular Nacional (2009, p.187) define los procesos de la siguiente manera:

- El proceso de Razonamiento y demostración implica desarrollar ideas, explorar fenómenos, justificar resultados, formular y analizar conjeturas matemáticas, expresar conclusiones e interrelaciones entre variables de los componentes del área y en diferentes contextos.

- El proceso de Comunicación matemática implica organizar y consolidar el pensamiento matemático para interpretar, representar (diagramas, gráficas y expresiones simbólicas) y expresar con coherencia y claridad las relaciones entre conceptos y variables matemáticas; comunicar argumentos y conocimientos adquiridos; reconocer conexiones entre conceptos matemáticos y aplicar la matemática a situaciones problemáticas reales.

- El proceso de Resolución de problemas implica que el estudiante manipule los objetos matemáticos, active su propia capacidad mental, ejercite su creatividad, reflexione y mejore su proceso de pensamiento al aplicar y adaptar diversas estrategias matemáticas en diferentes contextos. La capacidad para plantear y resolver situaciones problemáticas, dado el carácter integrador de este proceso, posibilita la interacción con las demás áreas curriculares coadyuvando al desarrollo de otras capacidades; asimismo, posibilita la conexión de las ideas matemáticas con intereses y experiencias del estudiante.

De lo expuesto, los procesos transversales del área de matemática en el razonamiento exigen desarrollar ideas, explorar fenómenos, justificar resultados, formular y analizar 
conjeturas matemáticas, en el proceso de comunicación matemática implica organizar y consolidar el pensamiento matemático para interpretar, representar y expresar. En el proceso de resolución de problemas implica que el estudiante manipule los objetos matemáticos, active su propia capacidad mental, ejercite su creatividad, reflexione y mejore su proceso de pensamiento al aplicar.

Las instituciones educativas buscan lograr en los estudiantes que sean competentes en el área de matemática buscan que los estudiantes logren resolver los problemas matemáticos, según las Rutas del Aprendizaje(2013), menciona que: La competencia matemática en la educación básica promueve el desarrollo de capacidades en los estudiantes, que se requieren para enfrentar una situación problemática en la vida cotidiana, alude sobre todo, a una situación eficaz en diferentes contextos reales a través de una serie de herramientas y acciones. Es decir, a una actuación que moviliza e integra actitud. (p. 19)

Por otro lado Según Gutiérrez (2008), considera que: La competencia matemática consiste en la habilidad para utilizar y relacionar los números, sus operaciones básicas, los símbolos y las formas de expresión y razonamiento matemático, tanto para producir e interpretar distintos tipos de información, como para ampliar el conocimiento sobre aspectos cuantitativos y espaciales de la realidad, y para resolver problemas relacionados con la vida cotidiana y con el mundo laboral. (p. 10)

La competencia matemática es entonces un saber actuar en un contexto particular, que promueve el desarrollo de capacidades en los estudiantes, que se requieren para enfrentar una situación problemática así mismo ampliar el conocimiento sobre aspectos cuantitativos y espaciales de la realidad, y para resolver problemas relacionados con la vida cotidiana.

La resolución de situaciones problemáticas es una competencia matemática importante que nos permite desarrollar capacidades matemáticas todas ellas existen de manera integrada y única en cada persona y se desarrollan en el aula, la escuela, la comunidad según las Rutas del aprendizaje (2013, pág. 22), menciona lo siguiente: Las capacidades matemáticas se despliegan a partir de las experiencias y expectativas de nuestros estudiantes, en situaciones problemáticas reales. Si ellos encuentran útil en su vida diaria los aprendizajes logrados, sentirán que la matemática tiene sentido y pertinencia. La propuesta pedagógica para el aprendizaje de la matemática toma en cuenta el desarrollo 
de seis capacidades matemáticas, consideradas esenciales para el uso de la matemática en la vida cotidiana. Éstas sustentan la competencia matemática de resolución de problemas y deben abordarse en todos los niveles y modalidades de la Educación Básica Regular. Estas seis capacidades son las siguientes: Matematizar. Representar, Comunicar, Elaborar estrategias, Utilizar expresiones simbólicas, Argumenta

Todas ellas están implicadas en cualquier situación problemática real, o matemática, si ellos encuentran útil en su vida diaria los aprendizajes logrados, sentirán que la matemática tiene sentido y pertinencia sustentando así el logro de la competencia.

En todo lugar y momento uno observa o escucha inconscientemente un problema para el cual según Echenique (2006, pág. 20), define el problemade la siguiente manera: Un problema es una situación que un individuo o grupo quiere o necesita resolver y para la cual no dispone, en principio, de un camino rápido y directo que le lleve a la solución; consecuentemente eso produce un bloqueo. Conlleva siempre un grado de dificultad apreciable, es un reto.

Así mismo Huánuco (2012, pág. 15)define el problema de la siguiente manera: "Problema es una dificultad cuestión o estado de desequilibrio que puede resolver o tratar de resolver mediante el pensamiento reflexivo, creativo y crítico".

Por consiguiente, un problema es una dificultad de una situación que un individuo o grupo quiere o necesita resolver mediante el pensamiento reflexivo, creativo y crítico, teniendo en cuenta los escenarios donde se plantea el problema.

El resolver problemas matemáticos es lograr una competencia en el que se pone de manifiesto la habilidad de las personas y el grado de desarrollo de las destrezas. Es la principal finalidad del área, entendida no solamente como la resolución de situaciones problemáticas propias de la vida cotidiana, sino también de las que no resulten tan familiares, según Echenique (2006), menciona que: "La resolución de problemas precisa de una planificación de las acciones a llevar a cabo, que ayuden a situar y utilizar adecuadamente los conocimientos adquiridos." (p. 17) Por lo tanto, el desarrollo de los problemas matemáticos precisa de una planificación de las acciones a llevar a cabo, utilizando el razonamiento, la capacidad de pensar y dar soluciones a problemas de la vida diaria. 
El matemático Polya (2002) menciona que la resolución de problemas matemáticos es: "Un proceso importante en la formación matemática de los niños para el desarrollo de la capacidad de reflexión.” (p. 66)

Así mismo el Ministerio de Educación (2004) lo define como: "Un problema es una situación nueva ante la cual hay que buscar y dar reflexivamente una respuesta coherente." (p. 07)

De este modo el problema matemático es una situación que permite desarrollar en los estudiantes las habilidades de razonamiento y creatividad en el espacio, ante la cual se busca un procedimiento importante para dar reflexivamente una respuesta coherente ante el problema planteado.

De entre todas las estrategias aplicables para el nivel de primaria es importante resaltar el juego según Gálvez (1996) define la estrategia de juego como: El juego es considerado como una de las extraordinarias estrategias que influencia decisiva en el desarrollo del niño, no solo porque facilita la interacción y construcción de los procesos Psicológicos Superiores, sino porque libera al niño de las coacciones a que se ve sometido, la formación de un conjunto de reglas y contenidos, la recuperación del verdadero sentido de la palabra escuela etc. (p. 391)

En conclusión, el juego es una estrategia que influencia en el desarrollo del niño, facilitando la interacción y construcción de su aprendizaje, por lo que se recupera el verdadero sentido de la palabra escuela dentro de los muchos contenidos que tradicionalmente se les impone.

Una gran actividad y un trabajo placentero para los niños es el juego, según Tineo (2006) define que: "El juego es una acción y una actividad voluntaria, realizada en ciertos límites fijados en tiempos y en lugares según una regla libremente aceptada, pero completamente imperiosa y provista de un fin” (p. 173).

El juego forma parte de todo niño y según Piaget (1932) leída en una publicación de Innovación y Experiencias Educativas(2008) menciona lo siguiente: "El juego forma parte de la función cognitiva general del sujeto, que él centra en la permanente búsqueda exploratoria y manipulativa. En los juegos la función cognitiva es, en alguna medida un proceso de mucho menos rigor adaptativo que en la actividad seria, y, en ese sentido, un proceso cognitivo imperfecto.” (p. 4) 
Por otro lado, el juego permite una relación entre dos individuos, para Bruner, en una publicación de Innovación y Experiencias Educativas(2008)define al juego de la siguiente manera: "El juego es un formato de actividad comunicativa entre iguales que les permite reestructurar continua y espontáneamente sus puntos de vista y sus conocimientos, mientras se divierten y gozan de la experiencia de estar juntos, e ir labrando el territorio para que nazcan y crezcan amistades interesantes.” (p. 5)

Por otra parte, el juego es una acción y una actividad voluntaria que forma parte de la función cognitiva general del sujeto, en un determinado tiempo, dentro de un lugar delimitado y reglas establecidas, les permite reestructurar continua y espontáneamente sus puntos de vista y sus conocimientos, mientras se divierten.

Siendo de gran importancia que el niño se sienta predispuesto en su aprendizaje es necesario el uso del juego por lo que Maestre (2009) menciona en la siguiente cita: El juego tiene un gran valor educativo, a través de él se hace más efectivo el aprendizaje, ya que el mundo del niño y la niña gira alrededor del juego. El juego desarrolla físicamente, crea y fomenta normas sociales y morales, además de ser agente de transmisión de ideas. La aplicación provechosa de los juegos posibilita el desarrollo biológico, psicológico y social del individuo. A nivel individual desenvuelve el lenguaje, despierta la capacidad de observación, la creatividad, la imaginación y la reflexión. La espontaneidad en la acción da rienda suelta a la libertad del sujeto, son olvidar, los beneficios físicos que proporciona, ya que pone en actividad todos los órganos del cuerpo. (p.2).

De este modo, el juego hace más efectivo el aprendizaje, ya que el mundo del estudiante gira alrededor del juego desarrollándose físicamente, crea y fomenta normas sociales y morales, de manera individual desenvuelve el lenguaje, despierta la capacidad de observación, la creatividad, la imaginación y la reflexión espontánea ante un problema o dificultad formando parte de su vida en un escenario determinado.

De todos los estudiantes dentro del aula, son pocos quienes tienen la capacidad de resolver problemas sin dificultades, o son muchos quienes no entienden la manera de dar solución a los problemas. El Ministerio de Educación (2001) menciona que la capacidad de resolución de problemas: "Requiere el uso de todas las capacidades intelectuales de quien aprende para encontrar alternativas viables ante una situación que es necesario resolver." (p. 84) 
Por otro lado, tener la capacidad de resolver problemas requiere de conocimientos básicos, según el Ministerio de Educación (2012) describe lo siguiente: "Se habla de resolver problemas, en lugar de simples ejercicios, cuando el estudiante logra solucionar una situación problemática dada, contextualizada o no, sin que se le haya indicado un procedimiento a seguir. Mediante estos desafíos, los alumnos experimentan, escogen o inventan y aplican diferentes estrategias, comparan diferentes vías de solución y evalúan las respuestas obtenidas y su pertinencia." (p.3)

En conclusión, la capacidad de resolución de problemas es la aplicación de todas las capacidades intelectuales, mediante estos desafíos, los estudiantes experimentan, escogen o inventan y aplican diferentes estrategias, comparan diferentes vías de solución y evalúan las respuestas obtenidas y su pertinencia.

Es de vital importancia que los estudiantes tengan la capacidad de resolver problemas en distintos escenarios de su vida cotidiana es así que el Ministerio de Educación (2013 p. 10) plantea lo siguiente: Asumimos el enfoque centrado en resolución de problemas o enfoque problémico como marco pedagógico para el desarrollo de las competencias y capacidades matemáticas, por dos razones:

La resolución de situaciones problemáticas es la actividad central de la matemática. Es el medio principal para establecer relaciones de funcionalidad matemática con la realidad cotidiana.

Este enfoque supone cambios pedagógicos y metodológicos muy significativos, pero sobre todo rompe con la tradicional manera de entender cómo se aprende la matemática, mostrando escenarios donde el niño se desenvuelve y planteando problemas de su vida cotidiana.

Se evidencia en muchos casos que la resolución de problemas está estrechamente relacionada con la creatividad, que algunos definen precisamente como la habilidad para generar nuevas ideas y solucionar todo tipo de problemas, dificultades y desafíos. Según Nieto (2004) puede resolverse de las siguientes maneras: Pensamiento lateral: Consiste en explorar alternativas inusuales o incluso aparentemente absurdas para resolver un problema. En otras palabras: evitar los caminos trillados, intentar lo que nadie ha intentado, ensayar percepciones y puntos de vista diferentes.

Principio de discontinuidad: La rutina suprime los estímulos necesarios para el acto creativo, por lo tanto, si experimenta un bloqueo temporal de su capacidad creadora 
interrumpa su programa cotidiano de actividades y haga algo diferente a lo acostumbrado. Vaya a dar un paseo por sitios que no conoce, ensaye una nueva receta de cocina, escuche música diferente a la que escucha habitualmente, lea un libro que no tenía pensado leer, asista a algún tipo de espectáculo diferente a sus favoritos.

Imitación: La mayor parte de los grandes artistas comienzan imitando a sus maestros. Más aún se ha llegado a armar, en parte en broma y en parte en serio, que Vla originalidad no es otra cosa que un plagio no detectado". En cualquier caso, es claro que la imitación puede ser un primer paso valido hacia la originalidad. En particular observe y no vacile en imitar las técnicas de resolución de problemas empleadas con éxito por sus compañeros, maestros o colegas.

Tormenta de cerebros (Brainstorming): Es una técnica desarrollada en el mundo de la publicidad, en el cual el éxito depende de la generación de nuevas y brillantes ideas. Para ello se reúne un grupo de personas y se les invita a expresar todas las ideas que se les ocurran en relación a un problema o tema planteado, sin importar lo estrafalarias o ridículas que parezcan. La evaluación y la crítica se posponen, esperando crear un clima estimulante que favorezca el surgimiento de algunas ideas realmente útiles. La utilidad de esta técnica es dudosa fuera de ciertos campos o situaciones muy específicas.

Se concluye que el ensayar percepciones y puntos de vista diferentes permite explorar alternativas inusuales, siendo importante el trabajo en grupo para que puedan dar ideas que se les ocurran en relación a un problema, pues la resolución de problemas no es un asunto puramente intelectual, en lo particular se debe de imitar las técnicas de resolución de problemas empleadas con éxito por otras personas (compañeros, profesor u otros), por otro lado, la rutina suprime los estímulos necesarios para el acto creativo de resolver situaciones problemáticas.

\section{ESTRATEGIAS METODOLÓGICAS O MATERIALES Y MÉTODOS}

El tipo de investigación es aplicada que tiene por finalidad de obtener conocimientos y solucionar problemas que se desarrolla mediante un proceso. Con un enfoque cuantitativo que se centra fundamentalmente en los aspectos observables y susceptibles de cuantificación de los fenómenos, sirviéndose de pruebas estadísticas.

La investigación corresponde a un diseño Pre-experimental, que tiende a establecer una relación de causa y efecto, con un grupo aleatorio al cual se le evaluará antes y después 
de la aplicación de la Estrategia LUDIPROBLEMAS para elevar la competencia de resolución de problemas matemáticos con la aplicación de Actividades vivenciales.

\section{EI Diseño adopta el siguiente esquema:}

\begin{tabular}{cccc}
\hline Grupo & Pre-test & $\begin{array}{c}\text { Variable } \\
\text { Independiente } \\
\text { (experiencia) }\end{array}$ & Post-test \\
\hline $\mathrm{G}$ & $\mathrm{O} 1$ & $\mathrm{X}$ & $\mathrm{O} 2$ \\
\hline
\end{tabular}

Dónde:

$\begin{array}{lll}\text { G } & : & \text { Grupo de aplicación } \\ \mathrm{X} & : & \text { Tratamiento Experimental } \\ \mathrm{O} 1 & : & \text { Prueba del Pre }- \text { Test } \\ \mathrm{O} 2 & : & \text { Prueba del Post }- \text { test }\end{array}$

\section{RESULTADOS Y DISCUSIÓN}

A continuación, se presenta los cuadros y gráficos estadísticos con su correspondiente análisis e interpretación.

\section{Tabla $\mathbf{N}^{\circ} 01$}

Resultados de la comparación entre la prueba de pre test y la prueba de post test de la competencia de resolución de problemas matemáticos

\begin{tabular}{|c|c|c|c|c|c|}
\hline \multirow{2}{*}{$\begin{array}{c}\text { Escala de calificación } \\
\text { cualitativa }\end{array}$} & \multicolumn{2}{|c|}{$\begin{array}{l}\text { Prueba } \\
\text { pre test }\end{array}$} & \multicolumn{2}{|c|}{$\begin{array}{c}\text { Prueba } \\
\text { post test }\end{array}$} & \multirow{2}{*}{ Estadística } \\
\hline & $\mathbf{F}$ & $\%$ & $\mathbf{F}$ & $\%$ & \\
\hline $\begin{array}{l}\text { AD } \\
\text { Logro Destacado }\end{array}$ & 0 & $0 \%$ & 7 & $29 \%$ & Pre test \\
\hline $\begin{array}{l}\text { A } \\
\text { Logro Previsto }\end{array}$ & 1 & $4 \%$ & 9 & $38 \%$ & $\begin{array}{l}\bar{X}=8,6 \\
\mathrm{~S}=2,7\end{array}$ \\
\hline B & 2 & $8 \%$ & 6 & $25 \%$ & $\begin{array}{l}\text { Post test } \\
\bar{X}=15,9\end{array}$ \\
\hline $\begin{array}{l}\mathrm{C} \\
\text { En inicio }\end{array}$ & 21 & $88 \%$ & 2 & $8 \%$ & $\mathrm{~S}=3,3$ \\
\hline Total & 24 & $100 \%$ & 24 & $100 \%$ & \\
\hline
\end{tabular}

Fuente: Prueba Pre test y Post Test aplicados a los estudiantes. 
La tabla $\mathrm{N}^{\circ} 01$ muestra los resultados comparativos entra la Prueba Pre Test y la Prueba Post Test aplicados en los estudiantes, siendo el grupo de aplicación, evidenciándose los siguientes resultados.

Antes de la aplicación de la Estrategia LUDIPROBLEMAS los estudiantes obtuvieron un promedio de 8,6 puntos, encontrándose en el nivel de inicio. Sin embargo, después de la aplicación de la experiencia, obtuvieron 15,9 puntos como promedio ubicándose en los niveles de Logro Previsto.

En conclusión, La Estrategia LUDIPROBLEMAS permite el desarrollo de la competencia de la resolución de problemas con aplicación de las actividades en el grupo de aplicación mostrando un incremento de 7,3 puntos.

\section{CONCLUSIÓN O CONSIDERACIONES FINALES}

1. Se concluye que el $88 \%$ de los estudiantes del grupo de aplicación antes de la Estrategia LUDIPROBLEMAS, se encontraban en el nivel de inicio con respeto al desarrollo de la competencia de la resolución de problemas matemáticos. Los cuales se evidencian a través de la prueba de Pre Test.

2. La aplicación de la Estrategia LUDIPROBLEMAS a través de las actividades para la comprensión y resolución de problemas, permitió superar las dificultades en la comprensión de enunciados, el dominio de las operaciones básicas y con ello el desarrollo de la competencia de resolución de problemas.

3. Se concluye que el $67 \%$ de los estudiantes del grupo de aplicación después de la experiencia se encuentra en un nivel de logro previsto en el desarrollo de la competencia de resolución de problemas con aplicación de las actividades de la estrategia LUDIPROBLEMAS.

4. Se afirma que el $29 \%$ de los estudiantes obtuvieron el logro destacado después de la aplicación de la Estrategia LUDIPROBLEMAS desarrollando así la competencia de la resolución de problemas.

5. La aplicación de la Estrategia LUDIPROBLEMAS permite el desarrollo de la competencia de resolución de problemas con aplicación de las actividades aplicadas en los estudiantes.

\section{REFERENCIAS BIBLIOGRAFICAS}

Acevedo Caicero, M. M. (2007). Fundamentación Conceptual Área de Matemáticas. Bogotá: s.e. 
Borda Soaquita, W. (2007). Aprendizaje esratégico. Tacna. s/e

Callao, D. R. (2012). "Estrategias didácticas y fichas de trabajo para la resolución de problemas aditivos". Callao: s/e.

Echenique Urdiain, I. (2006). Matemática resolución de Problemas. Navarra: Castuera.

Gálvez Vásquez, J. (1996). Métodos y Técnicas de Aprendizaje.

Gutierréz Ocerín, L. y. (2008). Las competencias básicas en las áreas de Matemáticas. Cantrabia: Consejería de Educación de Cántabria.

Huánuco Torres, A. (2012). Aplicación de estrategia para resolver problemas de cambio en los niños y niñas de primer grado "E" de la Institución Educativa Nº 6048 Jorge Basadre. Lima.

Llanos Vela, J. E. (1957). "500 juegos escolares". Arequipa: Jelive.

Maestre Castro, A. B. (febrero de 2009). El Juego: una gran estrategia educativa. Recuperado el 26 de junio de 2013, de El Juego: una gran estrategia educativa: http://www.csi-

csif.es/andalucia/modules/mod_ense/revista/pdf/Numero_15/ANA\%20BELEN_ MAESTRE_2.pdf

Malaspina Jurado, U. V. (2008). "Intuición y rigor en la resolución de problemas de optimización. Un análisis desde el enfoque ontosemiótico de la cognición e instrucción matemática". Lima: s/e.

Manolo Alcalá, J. M. (2006). Matemáticas re-creativas. Venezuela: GRAO, de IRIF, S.L.

Miguel, G. (2007). Enseñanza de la ciencia y la matemática.Iberioamericana de educación, 21.

Ministerio de Educación. (2004). Guía para el desarrollo de capacidades. Lima: Ministerio de Educación.

Ministerio de Educación. (2013). Hacer uso de saberes matemáticos para afrontar desafios diversos. Lima: Ministerio de Educación.

Ministerio de Educación. (2006). Orientación para el trabajo pedagógico de matemática. Lima: Firmat S.A.C.

Ministerio de Educación República del Perú. (2005). Evaluación Nacional del Rendimiento Estudiantil. Lima - Perú: Ministerio de Educación.

Nieto Said, J. H. (2004). Resolución de Problemas Matemáticos. Maracaibo: s/e.

Paenza, A. (2012). Matemática para todos. Buenos Aires: Printing Books S.A. 
Programa de Educación Logros de Aprendizaje. (212). PELA TALLER DE ACTUALIZACIÓN. Tacna - Perú: s.e.

Rutas de Aprendizaje. (2013). Hacer uso de los Saberes Matemáticos para afrontar los desafios diversos. Lima: Ministerio de Educación.

Tineo Campos, L. (2006). Eduque con juegos. Lima: Ediciones y representaciones B. Honorio. Págs 173. 